\title{
FORECASTING MESOSCALE PRECIPITATION USING THE MM5 MODEL WITH THE FOUR-DIMENSIONAL DATA ASSIMILATION (FDDA) TECHNIQUE
}

\author{
Y. YAMAZAKI* \\ M. D. M. ORGAZ
}

University of Aveiro, Department of Physics

Campus Santiago

3810-193 Aveiro, Portugal
Selected from papers presented in $9^{\text {th }}$ International Conference on Environmental Science and Technology (9CEST2005)

1-3 September 2005, Rhodes island, Greece *to whom all correspondence should be addressed: e-mail: yamasaki@fis.ua.pt

\begin{abstract}
The two basic forms of multi-scale data assimilation procedures (FDDA), based on Newtonian relaxation, of analysis and observations nudging have been applied for precipitation event period occurred over Portugal during summer season, using the Fifth Generation Mesoscale Model (MM5) developed and maintained by the Pennsylvania State University and National Center for Atmospheric Research (PSU/NCAR). The model has been configured for three nested grid domains covering part of the Eastern part of North Atlantic region evolving the Portugal, with 35 vertical levels, from surface up to 100 $\mathrm{hPa}$ top level. The model forecasting have been conducted employing daily available data from surface observational network, radio-sounding from Lisbon/Portugal and NOAA-16 polar orbiting satellite retrieved vertical profiles data. The three integration domains of MM5 model have been processed using, as boundary and first guess fields, the global atmospheric forecast NCEP-NWS/AVN model data gathered through the Unidata Local Data Manager (LDM)/Unidata Internet Data Distribution (IDD) system. All daily forecasting, with FDDA and with no FDDA, have been run for 60 hours forecast, with 30 minutes interval model data output to provide enough timely detailed results. The FDDA analysis presented a quite reasonable data ingesting volume of almost all available satellite data, with the exception of humidity data retrieved for high levels, above around $500 \mathrm{hPa}$. The obtained results indicate that, even using weak FDDA constraint coefficient values, presents a significant improvement in the numerical prognosis in the precipitation field, on both space and time integration levels. The results also presented an enhancement of the physics of the convective mesoscale system development, particularly over mountain region, indicating that it would be interesting to conduct an experiment with a dense data collecting platform coverage focused on events which occur in some prevailing mountain region of Portugal.
\end{abstract}

KEYWORDS: Mesoscale, Forecast, MM5, FDDA

\section{INTRODUCTION}

The importance of having an accurate initial state of the atmosphere for the success of numerical weather forecasting is nowadays quite well known following the recognizance that numerical models are very sensitive to small perturbations in the initial conditions. In fact, Best [1] showed that analysis errors could have serious effect on numerical prognoses, even including very small differences in the $500 \mathrm{hPa}$ analyses fields, using a single barotropic model, produces different prognoses that diverge as the forecast length increases. The slight variations in the initial wind field sensitivity study conducted by Huo [2] also show the importance of using accurate initial conditions to simulate important mesoscale features associated with Super-storms. In a more recent studies, according 
with Spencer and Stensrud [3], the improvement of initial conditions leads to a "significant improvement" in the precipitation field. Stensrud and Fritsch [4] also showed that simulations of the accuracy and evolution of events, associated with mesoscale convective systems (MCS's), are highly sensitive to differences in the techniques used to create the initial conditions.

Several factors are responsible for the impossibility of obtaining the perfect desirable initial state and conditions of the atmosphere. The data voids and insufficient data density coverage, observational errors, and analysis errors are among others the crucial factors since the model itself has direct influence only on the latter one. That explains why there are a variety of techniques available for mapping observations onto a grid. Even so, differences in model analysis, or a single and small change within a particular analysis procedure, can lead to a significant changes in the forecasts for a given set of observations.

Among several procedures, currently used by modelers for mapping observations onto a grid ([5], [[6], [7], [8], [9], [10]) the four-dimensional data assimilation (FDDA) is a suitable one to incorporate the asynoptic data into a forecast model. The Newtonian relaxation ("nudging"), which is one type of FDDA technique, adds an extra term to the prediction equation(s) in order to keep the model solution close to the observations. The second type of FDDA employs variational techniques which iteratively adjust the model forecast to the observations, by changing the initial and boundary conditions [11]. The variational technique was developed by Sasaki [12] and is a method for adjusting an objective analysis, by a minimum amount, necessary to satisfy certain dynamical constraints [13].

The basic form of multi-scale data assimilation procedures (FDDA), based on Newtonian relaxation, of analysis and observations nudging have been applied for a precipitation event occurred over Portugal during summer season. The procedures have been selected to include all observation data available, especially the asynoptic satellite data in the analysis. The precipitation event occurred during 2004, October 25-28 period over Portugal mainland have been selected for discussion. The mesoscale model forecasting has been processed employing both TOVS data from NOAA-16, as well as the Portugal automatic meteorological observation network (EMA) and upper air radio-sounding data.

\section{MESOSCALE MODEL DESCRIPTION, SETTINGS AND SIMULATION}

The Fifth Generation Mesoscale Model (MM5) developed and maintained by the Pennsylvania State University and National Center for Atmospheric Research (PSU/NCAR) is one of the most widely used public-domain prognostic mesoscale model. It has been developed at Penn State and NCAR, as a community mesoscale model with contributions from users worldwide, and detailed description of it is covered in [14] and [15] and also available at http://www.mmm.ucar.edu/mm5. The model is also supported by pre- and post-processing programs, collectively referred as MM5 modeling system, which facilitate the development of various model inputs, and the model output analysis.

The PSU/NCAR MM5 is a limited-area, terrain-following (sigma-coordinate), prognostic meteorological model, which solves the full suite of non-hydrostatic prognostic primitive equations for the three-dimensional wind, temperature, water (in all phases), and pressure fields. The model, which can run with multiple one-way or two-way nested grids, to resolve a range of atmospheric processes and circulations on spatial scales ranging from one to several thousands of kilometers, was configured for three nested grid domains (hereafter designated as D1, D2 and D3) as presented in Figure 1. The model equations are solved horizontally on an Arakawa-B grid structure defined, in the present simulation, on Lambert conformal conic projection centered on $40^{\circ} \mathrm{N}$ Latitude and $12^{\circ} \mathrm{W}$ Longitude. The vertical coordinate is a terrain-following normalized pressure coordinate, referred to as a "sigma", and have been configured with 35 vertical levels to resolve the troposphere and lower stratosphere up to $100 \mathrm{hPa}$. Other settings of the model are presented in the Table 1. The Figure 2 shows the terrain height of D3 and a East-West 
and North-South crossing lines over which the vertical cross sections of some variables have been analyzed.

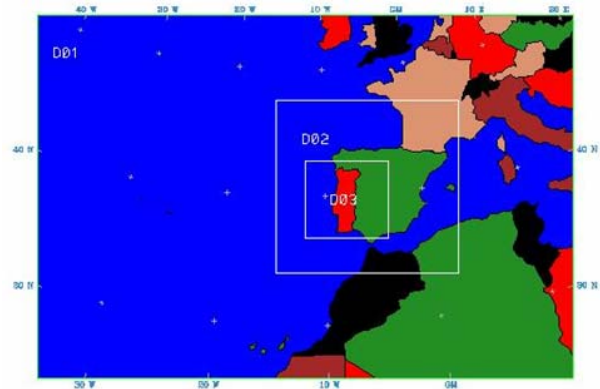

Figure 1. MM5 Domains

\begin{tabular}{||l|c|c|c|}
\hline Table 1. - MM5 Settings \\
\hline MM5 - Model domains & D1 & D2 & D3 \\
\hline Horizontal Resolution ( km ) & 81 & 27 & 9 \\
\hline \#Grig points East-West & 57 & 58 & 79 \\
\hline \#Grid points North-South & 39 & 55 & 73 \\
\hline Tpography resolution ( km) & 56 & 19 & 9 \\
\hline Time step (seconds) & 240 & 80 & 26,6 \\
\hline
\end{tabular}

The MM5 system Version 3.6 is highly modular and facilitates the interchange of physics and data assimilation options.

Among the several physical selection setting options, the one related to the humidity microphysics and cumulus parameterization are the ones mostly explored, at least when the main objective is related to precipitation forecasting [16]. In this respect, the cumulus parameterization selected has been the one which presented the best results on study already conducted for Portugal region [17]: Kain-Fritch 2 for D1 and D2 and Grell for D3. The explicit schemes of single ice of Dudhia, for D1 and D2; and Reisner mixed phase for D3 have been also pre-established. Details of these parameterizations are well documented in [15] and other parameters, as well as a detailed description are presented in [18].

The multi-scale data assimilation procedures (FDDA) have been applied for a precipitation event occurred over Portugal during the 2004, October 25-28 period. They have been processed employing both TOVS as well as the Portugal upper radio-sounding station and automatic meteorological observation network (EMA) data sources. The Figure 3 shows the total water vapor content in the atmosphere over the points on which the temperature and humidity vertical profiles data was also available for the FDDA model assimilation procedures in this particular Heliosynchronous NOAA-16 orbit. Other TOVS data coverage available for the present simulation has been from 13:35 UTC October 25; 00:14, 03:34, 13:24 and 15:08 UTC of October 26; 03:23, 14:56 UTC of October 27 and from 03:11, 014:44 UTC of October 28. The surface pressure, temperature and wind data from a total of 86 ground station of EMA have been also included in the FDAA.

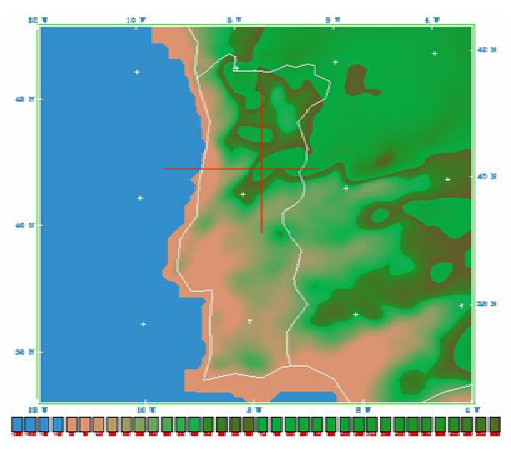

Figure 2. D3 Terrain height

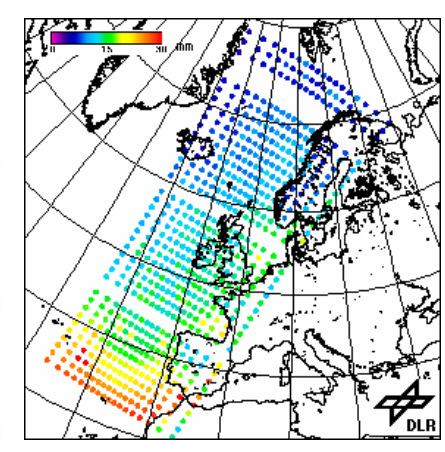

Figure 3. Total water vapor NOAA16

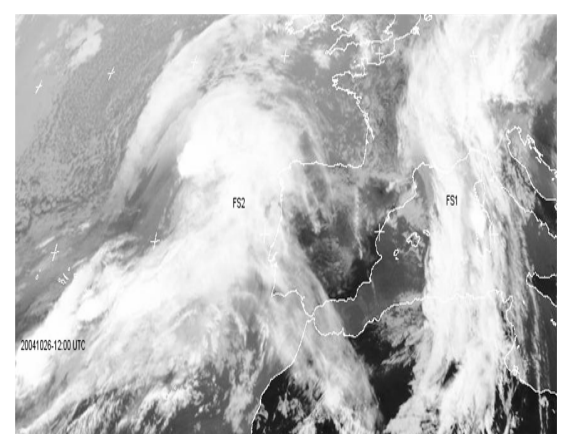

Figure 4. Meteosat IR-261004 12:00UTC 
The forecasts have been simulated for the three one-way nesting MM5 integration domains using the 6 hourly NCEP-NWS/GFS global atmospheric forecast model data as boundary and first guess fields for the October 25-28 period. The simulations have been made applying FDDA only during the first day, of each 60 hours of model integration experiment.

\section{RESULTS}

The satellite image of October 26, 12:00 UTC, from METEOSAT presented in Figure 4 shows the frontal system FS1 and FS2 which passed through the Iberian Peninsula bringing the thunder showers over Portugal Mainland simulated with the MM5 model. The first of it, FS1 only developed after passing to Portugal, during morning dawns of October 26 , and did not produced any significant rain over Portugal. On the other side FS2 developed, mostly during night time and particularly over the Northern sector of Portugal, and was the responsible for the rain, measured by the EMA's stations, as the 24 hours accumulated precipitation presented in Figure 5. It is worth to mention that Barne's type of interpolation have been used to plot the rainfall field, without any data void procedure applied to suspicious observed data, which probably explains the lack or rain in some region of the Central region of Portugal, or probably the excess of precipitation registered over other regions; opposing to model simulation presentation to be shown.

It is clearly shown that almost all MM5 simulations, initialized on day 25 , present a quite consistent rainfall distribution, irrespective to the amount of it, for the occurrence between 23:00 - 24:00 UTC of October 26. The no FDDA run, as presented in Figure 5-b shows less precipitation compared to the other runs. The use of surface data observations from EMA, as well as a single radio-sounding station data (Lisbon) in the FDDA produces more rain (Figure 5-c). The Figure 5-d shows the rainfall distribution when all available data, including surface, radio-sounding and TOVS data have been used in the FDDA.

The vertical cross sections along the lines presented in Figure 2 of the circulation vector, equivalent potential temperature and Potential Vorticity are presented in Figure 5 a-d. As it shows, the comparison between no $\operatorname{FDDA}(a, c)$ presents differing configurations on all these fields. The potential vorticity over the mountain region are too enhanced in noFDDA run case and although not presented here, the pre-storm phase development presented much more enhanced vertical velocity field along the mountain region in the FDDA run as compared with no-FDDA case.

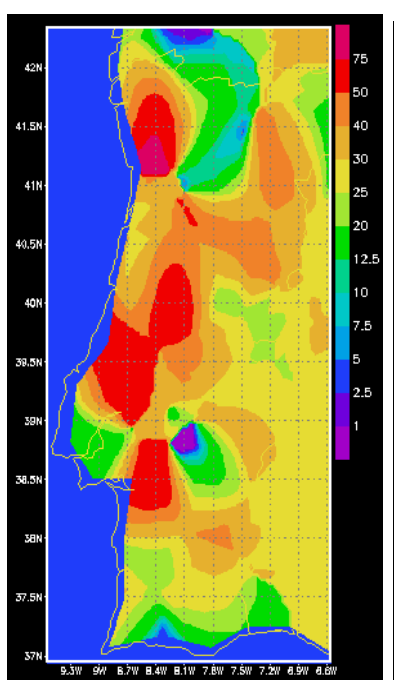

a

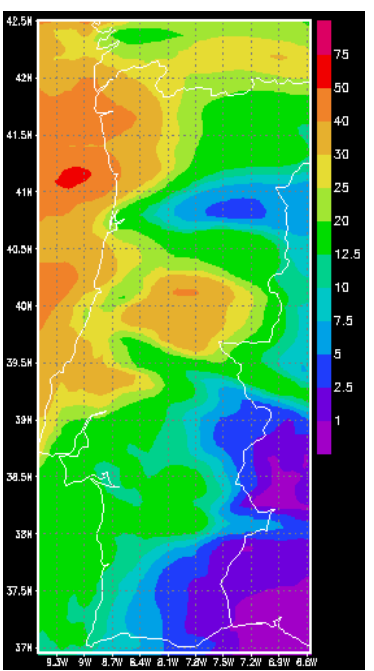

b

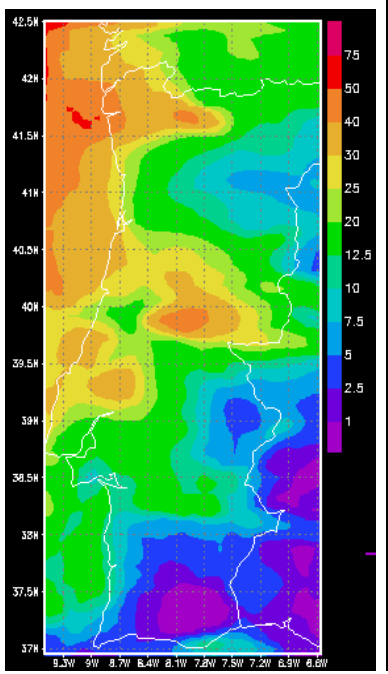

C

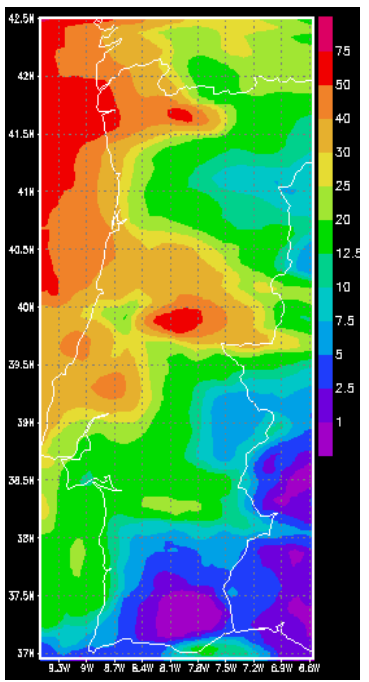

d

Figure 5. Rainfall distribution for October, 26. a- Observation; b-MM5 no FDDA; c- MM5 with surface FDDA; d- MM5 with full FDDA 


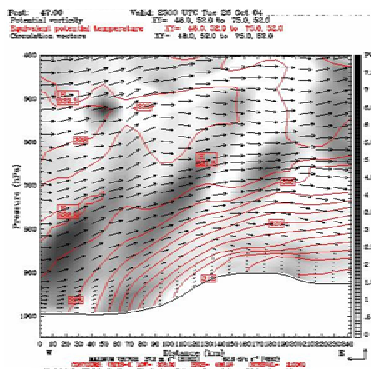

a

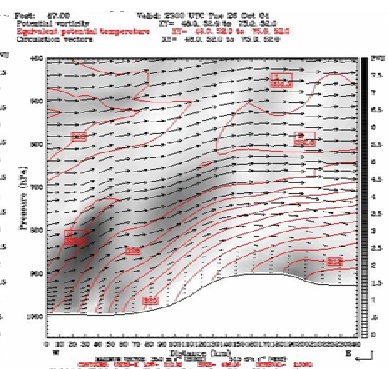

b

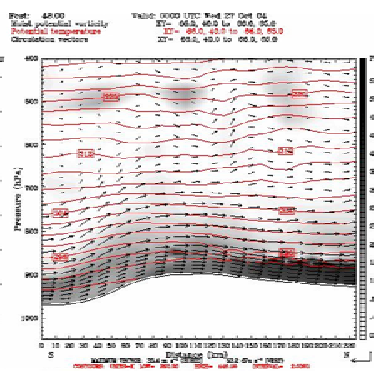

C

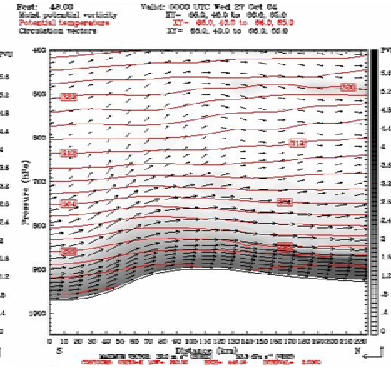

d

Figure 6. Potential Vorticity, Equivalent Potential Temperature and Circulation Vector Vertical cross sections for October : 23:00 UTC (a,b) over West-East direction and 24:00 UTC (c,d) over South-North direction

\section{CONCLUSIONS}

The basic form of multi-scale data assimilation procedures (FDDA), based on Newtonian relaxation, of analysis and observations nudging applied, for a precipitation event occurrence analysis over Portugal show that wind field over mountain region, as well as the potential vorticity, presents an enhancement when FDDA is applied, as compared with the case without it, especially when frontal system evolution occurs.

The results of precipitation analysis indicate that, even using weak constraint coefficient values, FDDA makes a significant improvement in the numerical prognosis in this variable distribution field, on both space and time integration levels.

Although there are a lack of a good space and time distributed surface data observational network, in particular over the Atlantic Ocean side of the Inland Portugal; to provide reliable data, which is a problem to be solved to allow an exhaustive comparison with observations, the conducted experiments presents the real potential of satellite data to improve the mesoscale model forecast.

\section{ACKNOWLEDGEMENT}

The research has been supported by Science and Technology Foundation (FCT) under grantFCT/REEQ946/2001, as well all first author's support - FCT/BD/19284.

The authors thank to National Meteorological Institute (IM) of Portugal and to the German Aerospace Center (DLR)/ German Remote Sensing Data Center for providing the data.

\section{REFERENCES}

1. Best, W.H. (1956) Differences in Numerical Prognoses Resulting from differences in Analyses, Tellus, 8, 351-356.

2. Huo, Z., Zhang D-L, Gyakum J., and Staniforth A. (1995) A Diagnostic Analysis of the Superstorm of March 1993. Mon. Wea. Rev., 123, 1740-1761.

3. Spencer, P.L, and Stensrud D.J. (1998) Simulating Flash Flood Events: Importance of the Subgrid Representation of Convection. Mon. Wea. Rev., 126, 2884-2912.

4. Stensrud, D.J., and Fritsch J.M. (1994) Mesoscale Convective Systems in Weakly Forced Large-Scale Environments. Part II: Generation of a Mesoscale Initial Condition. Mon. Wea. Rev., 122 (9), 2068-2083.

5. Bratseth, A.M. (1986) Statistical Interpolation by Means of Successive Corrections, Tellus, 38A, 439-447.

6. Le Dimet, F-X, and Talagrand O. (1986) Variational Algorithms for Analysis and Assimilation of Meteorological Observations: Theoretical Aspects, Tellus, 38A, 97-110.

7. McPherson, R.D. (1986), Mesoscale Meterology and Forecasting, Peter Ray, Ed., AMS, 151172. 
8. Parrish, D.F., and Derber J.C. (1992) The National Meteorological Center's Spectral Statistical-Interpolation Analysis System, Mon. Wea. Rev., 120, 1747-1763.

9. Sashegyi, K.D., Harms D.E., Madala R.V., and Raman S. (1993), Application of the Bratseth Scheme for the Analysis Of GALF, Data Using a Mesoscale Model, Mon. Wea. Rev., 121, 2331-2350.

10. Stensrud, D.J. and Fritsch J.M. (1994) Mesoscale Convective Systems in Weakly Forced Large-Scale Environments. Part III: Numerical Simulations and Implications for Operational Forecasting, Mon. Wea. Rev., 122 (9), 2084-2104.

11. Parsons, D.B. and Dudhia J. (1997), Observing System Simulation Experiments and Objective Analysis Tests in Support of the Goals of the Atmospheric Radiation Measurement Program, Mon. Wea. Rev., 125 (10), 2353-2381.

12. Sasaki, Y. K. (1958), An Objective Analysis Based on the Variational Method, J. Met. Soc. Japan, 36, 77-88.

13. Schlatter, T.W. (1988), Past and Present Trends in the Objective Analysis of Meteorological Data for Nowcasting and Numerical Forecasting', Preprints, Eighth Conference on Numerical Weather Prediction, Baltimore, Maryland, AMS, J9-J25

14. Dudhia, J. (1993) , A Nonhydrostatic Version of the Penn State-NCAR Mesoscale Model: Validation Tests and Simulation of an Atlantic Cyclone and Cold Front, Mon. Wea. Rev., 121, 1493-1513.

15. Grell, G., Dudia J. and Stauffer D. (1994), A Description of the Fifth-generation PennState/NCAR Mesoscale Model, NCAR Technical Report Note TN-398, National Center for Atmospheric Research, Boulder Colorado, US.

16. Chien F.-C., Jou B.J.-D., Lim P.-L., Hong P.-S. (2003) , A Real-time MM5/WRF Forecasting System in Taiwan, Thirteenth PSU/NCAR Mesoscale Model User's Workshop - NCAR, June 10-11, 2003.

17. Yamazaki Y. and Orgaz M.D.M. (2003), MM5 Rainfall and Cumulus Parameterizations Sensitivity Studies, Thirteenth PSU/NCAR Mesoscale Model User's Workshop - NCAR, June 10-11, 2003.

18. Dudhia, J. and Bresch J. F. (2002) A Global Version of the PSU-NCAR Mesoscale Model, Mon. Wea. Rev., 130 (12), 2989-3007. 